\title{
Neural mechanisms underlying the induction and relief of perceptual curiosity
}

\section{Marieke Jepma ${ }^{1,2}{ }^{*}$, Rinus G. Verdonschot ${ }^{1,2,3,4}$, Henk van Steenbergen ${ }^{1,2}$, Serge A. R. B. Rombouts ${ }^{1,2,5}$ and Sander Nieuwenhuis ${ }^{1,2}$}

\author{
Institute of Psychology, Leiden University, Leiden, Netherlands \\ ${ }^{2}$ Leiden Institute for Brain and Cognition (LIBC), Leiden, Netherlands \\ ${ }^{3}$ Leiden University Centre for Linguistics, Leiden, Netherlands \\ ${ }^{4}$ Graduate School of Languages and Cultures, Nagoya University, Nagoya, Japan \\ ${ }^{5}$ Department of Radiology, Leiden University Medical Center, Leiden, Netherlands
}

\section{Edited by:}

Antonella Gasbarri, University of I'Aquila, Italy

Reviewed by:

Bianca Wittmann, Justus Liebig

University Giessen, Germany

Jordan Litman, University of South

Florida, USA

\section{*Correspondence:}

Marieke Jepma, Department of

Psychology and Neuroscience,

University of Colorado Boulder,

Muenzinger D244, 345 UCB,

Boulder, CO 80309-0345, USA.

e-mail: marieke.jepma@colorado.edu
Curiosity is one of the most basic biological drives in both animals and humans, and has been identified as a key motive for learning and discovery. Despite the importance of curiosity and related behaviors, the topic has been largely neglected in human neuroscience; hence little is known about the neurobiological mechanisms underlying curiosity. We used functional magnetic resonance imaging (fMRI) to investigate what happens in our brain during the induction and subsequent relief of perceptual curiosity. Our core findings were that (1) the induction of perceptual curiosity, through the presentation of ambiguous visual input, activated the anterior insula and anterior cingulate cortex (ACC), brain regions sensitive to conflict and arousal; (2) the relief of perceptual curiosity, through visual disambiguation, activated regions of the striatum that have been related to reward processing; and (3) the relief of perceptual curiosity was associated with hippocampal activation and enhanced incidental memory. These findings provide the first demonstration of the neural basis of human perceptual curiosity. Our results provide neurobiological support for a classic psychological theory of curiosity, which holds that curiosity is an aversive condition of increased arousal whose termination is rewarding and facilitates memory.

Keywords: curiosity, fMRI, arousal, memory, reward processing

\section{INTRODUCTION}

Curiosity is a basic biological drive in both animals and humans, and has been identified as a key motive for learning and discovery. In the 1950s and 1960s, curiosity and related behaviors were topics of intense investigation among experimental psychologists, resulting in an extensive theoretical framework for understanding curiosity and related behaviors (e.g., Berlyne, 1954, 1960, 1966; Loewenstein, 1994). However, despite the importance of curiosity in many aspects of behavior, little is known about the neurobiological mechanisms underlying curiosity. In the present study, we used functional magnetic resonance imaging (fMRI) to test specific predictions of a classic psychological theory of curiosity, developed by Berlyne (1954). According to this theory, curiosity evoked by ambiguous, complex, or conflicting stimuli is an aversive condition associated with increased levels of arousal. The theory further holds that termination of this condition, through access to relevant information, is rewarding and promotes learning.

We focused on perceptual curiosity, the most basic type of curiosity that is found in animals as well as humans. One way to induce perceptual curiosity is to present subjects with blurred pictures. An early study using this method showed that blurred pictures evoked longer EEG desynchronization (alphawave blocking) than clear pictures, but only when the identity of the blurred pictures was unknown, which provides preliminary evidence that perceptual curiosity causes an increase in arousal (Berlyne and Borsa, 1968). Another experiment showed that participants actively preferred to view the clear version of a preceding blurred picture over viewing an unrelated clear picture (Nicki, 1970). Importantly, the preference for uncertainty reduction disappeared when participants knew the identity of the blurred picture. These findings are consistent with the idea that the reduction of perceptual curiosity is rewarding.

We used a modified version of the blurred pictures paradigm to investigate the neural underpinnings of both the induction and the subsequent relief of human perceptual curiosity. More specifically, we examined whether we could find support at the neural level for the main assumptions of Berlyne's theory. First, the assumption that curiosity is an aversive condition of increased arousal predicts that the induction of curiosity will produce activation in brain areas sensitive to autonomic arousal, conflict and other aversive states. The two brain regions that are typically activated by a broad range of aversive conditions (including pain, uncertainty, errors and disgust) are the anterior cingulate cortex (ACC; Ridderinkhof et al., 2004; Shackman et al., 2011) and the anterior insular cortex (AIC; Peyron et al., 2000; Singer et al., 2009); hence we predicted that perceptual curiosity would activate these regions. Second, the assumption that the reduction of curiosity is rewarding predicts that this will produce activation in brain regions involved in reward processing, such as the striatum. 
Third, the assumption that the reduction of curiosity promotes learning and memory predicts that uncertainty-reducing stimuli will be associated with enhanced memory performance and increased hippocampal activation.

\section{MATERIALS AND METHODS PARTICIPANTS}

Nineteen healthy volunteers participated (14 women and five men; aged $19-29$ years; mean age $=22.8$ years; $S D=2.4$ ), in return for $€ 25$. All participants gave written consent before participation, and the study was approved by the medical ethics committee of the Leiden University Medical Center. All participants had normal or corrected-to-normal vision and reported to be right-handed.

\section{TASK DESIGN}

Participants were scanned while they viewed sequences of two pictures of common objects, in a passive-viewing task. To manipulate the induction and reduction of perceptual uncertainty, we used the following four combinations of clear and blurred pictures (Figure 1):

1. A blurred picture followed by its corresponding clear picture (B-C $\mathrm{C}_{\text {corresponding }}$ )

2. A blurred picture followed by an unrelated clear picture (B-C $\mathrm{C}_{\text {unrelated }}$ )

3. A clear picture followed by its corresponding blurred picture (C-B)

4. A clear picture followed by an identical picture (C-C).

Entropy, an information-theoretic measure of uncertainty, increases with the number of possible outcomes and with the nearness in likelihood of the different possible outcomes (Shannon, 1948). Berlyne (1965, p. 246) proposed that someone's subjective uncertainty about a specific stimulus or event (e.g., the identity of an object or the solution to a problem) depends in a

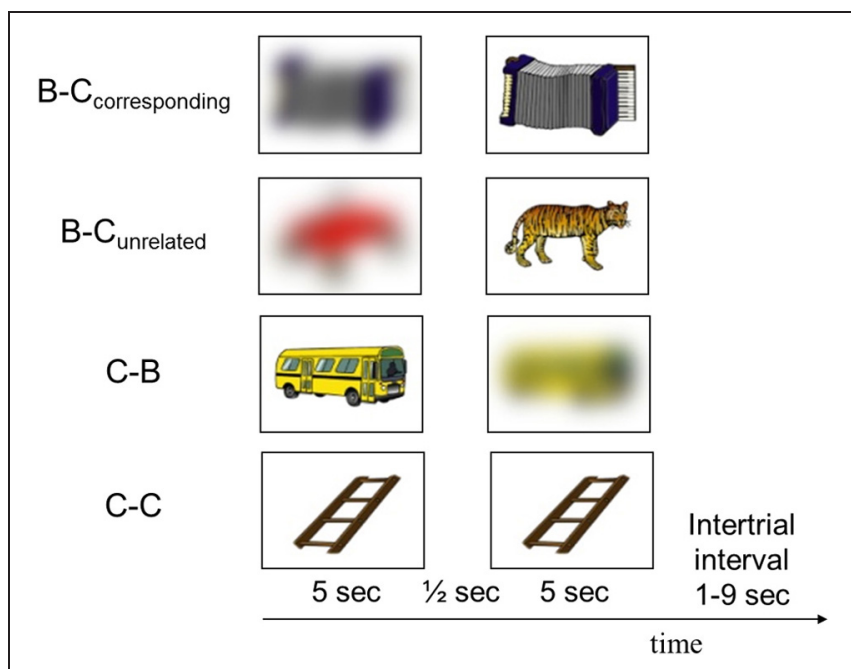

FIGURE 1 | Examples of pictures presented in each of the four conditions. The experiment consisted of 35 trials from each condition, presented in pseudorandom order. similar way on the number of alternative hypotheses, and the relative confidence placed in each hypothesis. A previous behavioral study using the blurred pictures paradigm has shown that subjective uncertainty (derived from the number of guesses regarding the picture's identity and the relative confidence placed in each guess) was maximal for pictures with an intermediate degree of blur (Nicki, 1970). That study also showed that participants' preference to see the corresponding clear version of a blurred picture was larger for pictures with an intermediate degree of blur than for pictures with either a very low or a very high degree of blur. Based on these findings, we only used blurred pictures with an intermediate degree of blur (i.e., with maximal subjective uncertainty).

All pictures were selected from Rossion and Pourtois' colored picture databank (Rossion and Pourtois, 2004). This databank is a set of 260 colored line drawings of objects, provided with norms for name agreement, image agreement, familiarity, and complexity ratings. We selected 140 pictures with perfect name agreement from this databank. The pictures had a resolution of $71 \mathrm{dpi}$, and were centered on a white rectangle of $197 \times 281$ pixels. We created a blurred version of each picture by means of Gaussian smoothing with a radius of 20-22 pixels (Adobe Photoshop 5.0; all pictures can be found at www.sandernieuwenhuis.nl/SOM). By reducing the picture's high-frequency components, Gaussian smoothing acts as a low-pass filter. Results from a behavioral pilot experiment with 49 participants indicated that the objects displayed in the blurred pictures could not be identified by the majority of the participants.

On each trial, a sequence of two pictures was presented. The pictures were projected onto a screen and viewed through a mirror attached to the head coil of the scanner. Each picture was presented for $5 \mathrm{~s}$ in the middle of the screen on a white background, and was surrounded by a black frame (visual angle $=18.5 \times 13.8^{\circ}$ ). The two pictures in a trial were separated by a $500 \mathrm{~ms}$ interval during which only the frame was presented. The intertrial interval varied between 1 and $9 \mathrm{~s}$ (uniform distribution). The experiment consisted of 35 trials from each of four conditions illustrated in Figure 1, presented in pseudorandom order. For the blurred pictures in the $\mathrm{B}-\mathrm{C}_{\text {unrelated }}$ condition we used blurred versions of 35 additional pictures from Rossion and Pourtois' databank (i.e., pictures of which the clear version was not used). The 140 clear pictures were presented in the same order for all participants. To exclude the possibility that differences between the conditions were caused by picture-specific effects, we divided the 140 clear pictures into four subsets of 35 pictures with comparable familiarity, complexity and imagery ratings (all $p s>0.86$ ) and alternated the coupling of the four picture subsets to the four conditions across participants according to a balanced Latin-square design.

The experiment was divided into five runs of 28 trials between which we stopped the scanner to verify that the participant was still attending to the pictures. Each run contained seven trials from each condition and lasted approximately $8 \mathrm{~min}$. Participants were not aware of the aim of the study; we told participants that the experiment was designed to investigate the brain activation associated with the perceptual processing of clear and blurred pictures, and informed them of the four possible ways in which clear and blurred pictures could be combined. 
After completing the experiment, participants were given an unexpected free-recall test outside the scanner; they were asked to type in the names of as many objects as they could recall from the pictures they had seen in the scanner. Subsequently, participants were asked to indicate, on a five-point scale ( $1=$ not at all; $5=$ very much), the degree to which they had (1) been curious about the blurred pictures; (2) tried to guess the identity of the objects depicted on the blurred pictures; (3) been disappointed when a blurred picture was not followed by the corresponding clear version; (4) recognized the objects depicted on the blurred pictures; and (5) tried to remember the pictures. Finally, participants completed the perceptual curiosity scale (Collins et al., 2004).

\section{IMAGE ACQUISITION}

Scanning was performed with a standard whole-head coil on a 3-T Philips Achieva MRI system (Best, The Netherlands). In each of the five functional runs, $210 \mathrm{~T} 2 *$-weighted whole-brain EPIs were acquired $(\mathrm{TR}=2.2 \mathrm{~s} ; \mathrm{TE}=30 \mathrm{~ms}$, flip angle $=80,38$ axial slices, $2.75 \times 2.75 \times 2.75 \mathrm{~mm}+10 \%$ interslice gap). In addition, a high-resolution EPI scan and a T1-weighted anatomical scan were obtained for registration purposes (EPI scan: TR $=2.2 \mathrm{~ms}$; $\mathrm{TE}=30 \mathrm{~ms}$, flip angle $=80^{\circ}, 84$ axial slices, $1.96 \times 1.96 \times 2 \mathrm{~mm}$; 3D T1-weighted scan: $\mathrm{TR}=9.7 \mathrm{~ms} ; \mathrm{TE}=4.6 \mathrm{~ms}$, flip angle $=8^{\circ}$, 140 axial slices, $0.88 \times 0.88 \times 1.2 \mathrm{~mm}$ ).

\section{IMAGE ANALYSIS}

MRI data analysis was carried out using FMRI Expert Analysis Tool (FEAT) version 5.98, which is part of FMRIB's Software Library (FSL; Smith et al., 2004). Image pre-processing consisted of motion correction (Jenkinson et al., 2002), non-brain removal (Smith, 2002), spatial smoothing using an $8 \mathrm{~mm}$ full-width at half-maximum (FWHM) Gaussian kernel, grand-mean intensity normalization of the entire $4 \mathrm{D}$ data set by a single multiplicative factor, and high-pass temporal filtering to remove low-frequency artifacts (Gaussian-weighted least-squares straight line fitting, with sigma $=100 \mathrm{~s}$ ). Functional scans were registered to highresolution EPI images, which were registered to T1 images, which were registered to standard MNI space (Jenkinson and Smith, 2001; Jenkinson et al., 2002).

The fMRI time series were analyzed using an event-related approach in the context of a general linear model with local autocorrelation correction (Woolrich et al., 2001). We constructed six explanatory variables of interest: two for the first picture in a trial (Blurred or Clear), and four for the second picture in a trial (Clear-corresponding, Clear-unrelated, Clear-double, or Blurred). Each explanatory variable was time-locked to the picture onset and had a duration of $5 \mathrm{~s}$ (i.e., the entire duration of the picture presentation). The hemodynamic response to each event was estimated by convolving each explanatory variable with a canonical hemodynamic response function and its temporal derivative. The model was high-pass-filtered (Gaussian-weighted least-squares straight line fitting, sigma $=100 \mathrm{~s}$ ).

For each run, in each participant, we assessed several contrasts (see Results section). The contrasts were combined across the five runs on a subject-by-subject basis using fixed-effects analyses (Beckmann et al., 2003; Woolrich et al., 2004). These second-level contrast images were submitted to third-level mixed-effects group analyses (Beckmann et al., 2003; Woolrich et al., 2004).

\section{Region-of-interest analyses}

We conducted region-of-interest (ROI) analyses to test the predicted AIC and ACC activation in response to the induction of perceptual uncertainty, and the predicted striatal and hippocampal activation in response to the relief of perceptual uncertainty. We used anatomical ROIs of the bilateral insular cortex, ACC (comprised of the anterior cingulate and paracingulate gyrus; Fornito et al., 2006), striatum (comprised of the caudate, putamen and nucleus accumbens), and hippocampus, as specified by the Harvard-Oxford subcortical structural atlas, and implemented in FSLView version 3.1.2. Only the voxels that were part of these areas with a probability of at least $50 \%$ were included in the ROIs. The statistical parametric images were thresholded using clusters determined by $Z>2.3$ and a cluster-corrected significance threshold of $p<0.05$ (Worsley, 2001). To further examine the hippocampal activation, we extracted the average time course of the hemodynamic response function in response to the second picture in each of the four conditions using PEATE (perl event-related average time course extraction), a companion tool to FSL (http://www.jonaskaplan.com/peate/peate-tk.html). Time courses were extracted from the hippocampal activation clusters of the curiosity-relief contrast (i.e., the regions with stronger activation in response to the second picture in the $\mathrm{B}-\mathrm{C}_{\text {corresponding }}$ condition than in response to the second picture in the $\mathrm{B}-\mathrm{C}_{\text {unrelated }}$ condition).

To examine whether individual differences in trait-perceptual curiosity and free-recall performance were predictive of individual differences in brain activation, we extracted each participant's peak $z$ value from the activation clusters of interest. We correlated these peak $z$ values with participants' scores on the perceptual curiosity questionnaire and with their free-recall performance. In addition, we computed the across-subject correlations between the peak $z$ values of the different activation clusters of interest.

\section{Disappointment median-split analysis}

To examine whether participants' rated disappointment when the identity of a blurred object was not revealed predicted the strength of their ACC/AIC activation in response to perceptual uncertainty, we divided all participants into two groups based on their disappointment ratings: nine participants reported a strong disappointment (ratings of 4 or 5 on a five-point scale) and the other ten participants reported less disappointment (ratings of 2 or 3 ). We used a $t$-test to determine whether the highdisappointment group showed stronger ACC/AIC activation in response to perceptual uncertainty than the low-disappointment group.

\section{Whole-brain analyses}

In addition to the ROI analyses, we conducted exploratory wholebrain analyses to examine the activation of brain areas outside our ROIs in response to induction and/or relief of perceptual uncertainty. In these analyses, statistical parametric images for each contrast were thresholded at $p<0.001$, with a minimum cluster size of $26 \mathrm{MNI}$ voxels $\left(208 \mathrm{~mm}^{3}\right)$. 
Table 1 | Participants' ratings of the degree to which they had been curious about the blurred pictures, recognized the blurred pictures, and had tried to remember the pictures (means \pm standard deviations).

I was curious about the blurred pictures $4.11 \pm 0.88$

I tried to guess the identity of the objects depicted in the $4.53 \pm 0.70$ blurred pictures

I was disappointed when a blurred picture was not followed by its clear version

I recognized the objects depicted in the blurred pictures $3.16 \pm 1.02$

I tried to remember the pictures

$2.79 \pm 0.92$

$1.74 \pm 0.73$

All ratings were on a scale from 1 (not at all) to 5 (very much).

\section{RESULTS}

We scanned 19 healthy participants while they viewed sequences of two pictures, in a passive-viewing task (Figure 1). Our task design resulted in the induction of perceptual uncertainty by the first picture on half of the trials (the $\mathrm{B}-\mathrm{C}_{\text {corresponding }}$ and $\mathrm{B}-\mathrm{C}_{\text {unrelated }}$ conditions), which was resolved by the second picture on half of these trials (the $\mathrm{B}-\mathrm{C}_{\text {corresponding }}$ condition). Participants' ratings after the scanning session indicated that they had indeed been curious about the blurred pictures (Table 1).

\section{FREE-RECALL PERFORMANCE}

The number of pictures that participants recalled in an unexpected free-recall test after the scan session was significantly affected by the condition in which the pictures had been presented $[F(3,54)=11.5, p<0.001]$. Participants recalled more pictures from the $\mathrm{B}-\mathrm{C}_{\text {corresponding }}$ condition (mean $=10.4, \mathrm{SD}=4.8$ ) than pictures from the $\mathrm{B}-\mathrm{C}_{\text {unrelated }}($ mean $=6.1, \mathrm{SD}=3.7), \mathrm{C}-\mathrm{C}$ (mean $=7.3, \mathrm{SD}=3.8)$ and $\mathrm{C}-\mathrm{B}($ mean $=8.0, \mathrm{SD}=4.4)$ conditions (all ps $<0.03$ ). Thus, incidental memory for uncertaintyreducing stimuli was enhanced. In addition, participants recalled fewer pictures from the $\mathrm{B}-\mathrm{C}_{\text {unrelated }}$ condition than from the $\mathrm{C}-\mathrm{C}$ and $\mathrm{C}-\mathrm{B}$ conditions $(p s<0.04)$. The number of pictures recalled from the $\mathrm{C}-\mathrm{C}$ and $\mathrm{C}-\mathrm{B}$ conditions did not differ $(p=0.28)$.

\section{BRAIN ACTIVATION ASSOCIATED WITH PERCEPTUAL UNCERTAINTY}

To examine the brain activation associated with perceptual uncertainty, we focused on the neural response to the first picture in each trial, and identified brain regions where activation was larger when the picture was blurred compared to clear. Our ROI analyses of the ACC and insular cortex revealed significant activation in the ACC (one cluster extending into both hemispheres) and the right AIC (Figure 2, upper panel; Table 2, upper part). Functional-connectivity studies have suggested that the AIC and AAC are part of a putative "salience network" (Seeley et al., 2007), which has been associated with autonomic arousal (Critchley, 2005) and various aversive emotional experiences (e.g., Craig et al., 1996; Ploghaus et al., 1999; Eisenberger et al., 2003).

The whole-brain analysis for this contrast also revealed activation in the bilateral AIC and ACC, as well as activation in regions of the inferior frontal gyrus, frontal pole, lingual gyrus, occipital pole and posterior cingulate gyrus (Table 3 ).

A whole-brain analysis (cluster-corrected, $p<0.05$ ) of the opposite contrast, which identified brain regions that were more

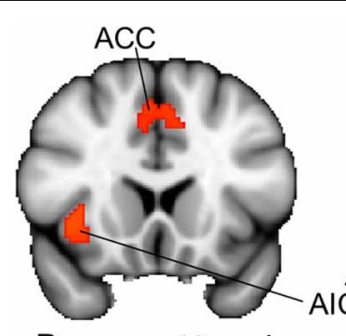

$\mathrm{R}$
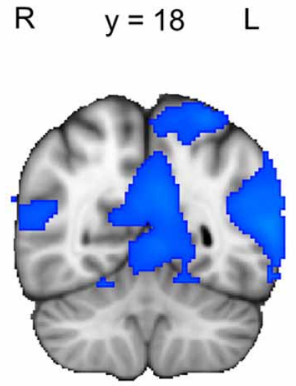

$\mathrm{R}$

$\mathrm{L}$
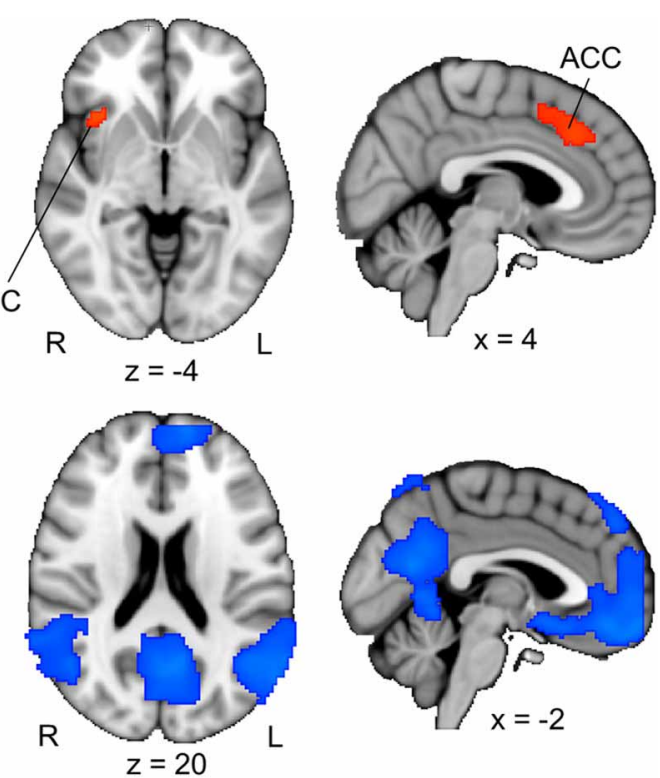

$y=-56$

FIGURE 2 | ACC/AIC activation associated with perceptual uncertainty. Upper panel: The colored regions were more active when the first picture in a trial was blurred (i.e., the first pictures in the $\mathrm{B}-\mathrm{C}_{\text {corresponding }}$ and $\mathrm{B}-\mathrm{C}_{\text {unrelated }}$ conditions) than when it was clear (i.e., the first pictures in the $\mathrm{C}-\mathrm{C}$ and $\mathrm{C}-\mathrm{B}$ conditions). $R=$ right; $L=$ left; $\mathrm{ACC}=$ anterior cingulate cortex; $\mathrm{AIC}=$ anterior insular cortex. The displayed activations are the cluster-corrected $Z$ statistic maps $(p<0.05)$ from the ACC/AIC $\mathrm{ROI}$ analyses. Lower panel: The colored regions were deactivated when the first picture in a trial was blurred compared to when it was clear. The displayed activations are whole-brain cluster-corrected $Z$ statistic maps $(p<0.05)$. All activations are overlaid onto the standard MNI brain. 
Table 2 | Activation clusters from the ROI analyses.

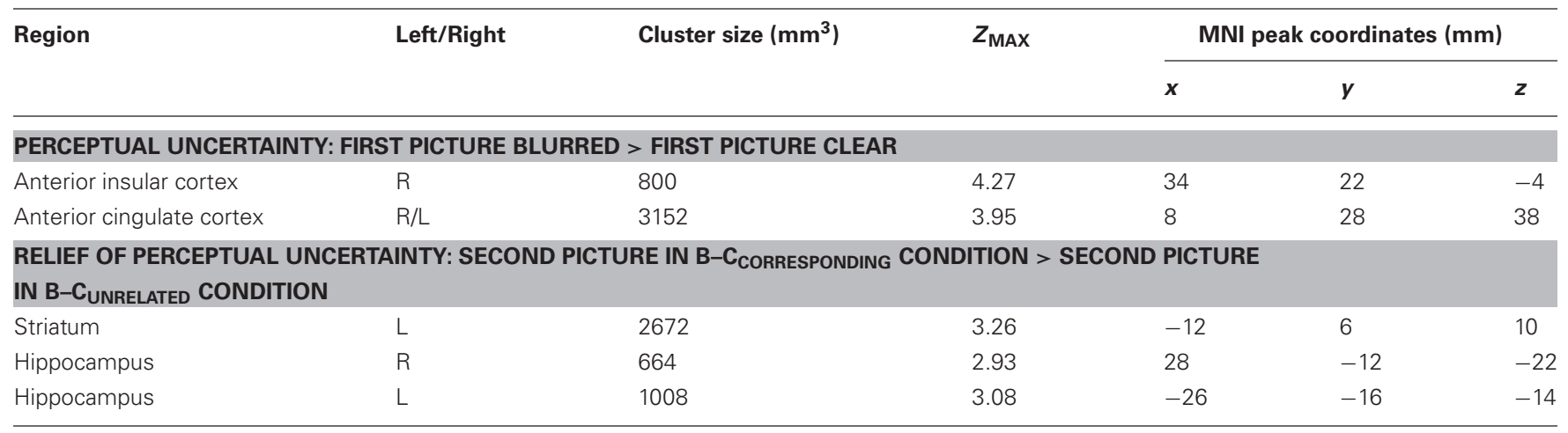

Table 3 | All brain regions that were activated in response to perceptual uncertainty (upper part) and in response to the relief of uncertainty (lower part).

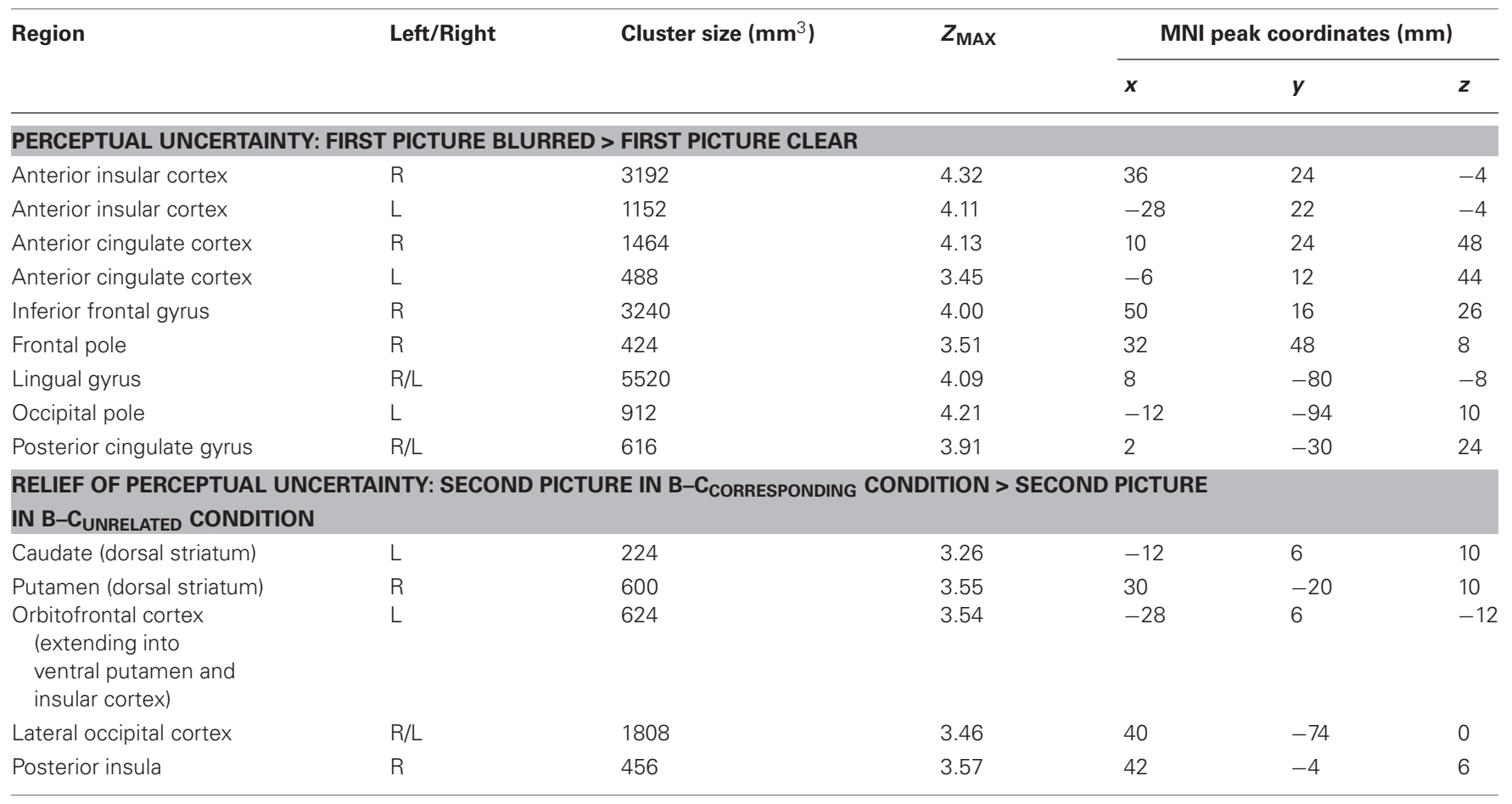

Data are thresholded at $p<0.001$ (uncorrected) with a minimum cluster size of 26 contiguous MNI voxels.

activated by clear pictures than by blurred pictures, revealed activation in a set of brain regions that have been associated with the "default-mode network" (Figure 2, lower panel). The default-mode network, which includes regions of the precuneus, posterior lateral parieto-occipital cortex and medial prefrontal cortex, is typically stronger activated during rest than during cognitive effort (e.g., Raichle et al., 2001). The relative deactivation of this network in response to blurred compared to clear pictures suggests that participants actively processed the blurred pictures. Consistent with this interpretation, participants indicated that they had been curious about the blurred pictures, had tried to guess the identities of the objects depicted in them, and had been rather disappointed when a blurred picture was not followed by its corresponding clear version (Table 1).

Several findings suggest that the AIC/ACC activation reflected a neural substrate of a negative arousal state associated with perceptual curiosity. First, the activated regions of the AIC and ACC closely overlap with areas that are typically activated in response to errors, negative feedback and other aversive events (Ullsperger et al., 2010). Second, the strength of participants' AIC activation was positively correlated with their trait curiosity as indexed by the perceptual curiosity questionnaire $(r=0.54$, $p=0.02$; Figure 3). Third, the participants who reported more disappointment when the identity of a blurred picture was not 


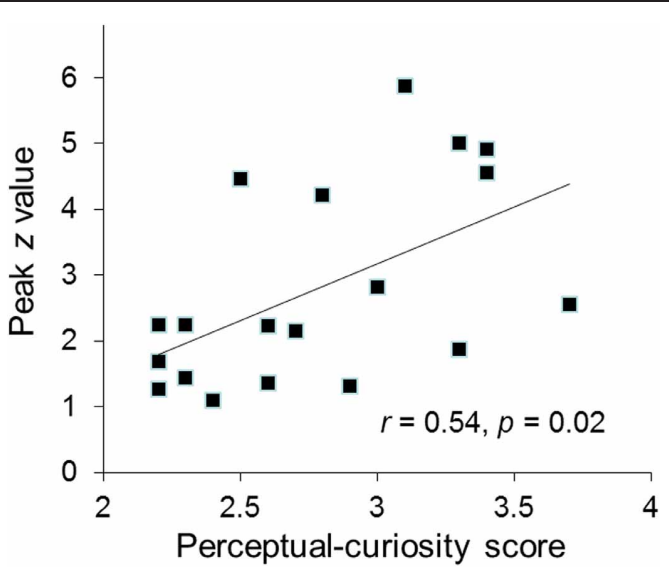

FIGURE 3 | The individual participants' peak activation ( $z$ value) for the perceptual-uncertainty contrast in the right AIC plotted against their perceptual curiosity score.

revealed showed stronger ACC activation than the participants who reported less disappointment $[t(17)=2.4, p=0.04$; see Section "Disappointment median-split analysis"].

Interestingly, the strength of participants' ACC activation associated with perceptual uncertainty was predictive of the number of pictures they later recalled from the $\mathrm{B}-\mathrm{C}_{\text {corresponding }}$ condition $(r=0.51, p=0.03$; there was also a trend for a positive correlation between the numbers of pictures recalled from the $\mathrm{B}-\mathrm{C}_{\text {corresponding }}$ condition and the AIC activation, $\left.p=0.075\right)$, but not of the number of pictures they recalled from the other conditions (all $p s>0.28$ ). This suggests that the uncertainty-related activation of the ACC contributed to the enhanced memory for stimuli that reduced this uncertainty.

\section{BRAIN ACTIVATION ASSOCIATED WITH THE RELIEF OF PERCEPTUAL UNCERTAINTY}

To examine the brain activation associated with the relief of perceptual uncertainty, we created a contrast that identified brain regions where activation was larger in response to the second picture in the $\mathrm{B}-\mathrm{C}_{\text {corresponding }}$ condition than in response to the second picture in the $\mathrm{B}-\mathrm{C}_{\text {unrelated }}$ condition. Our ROI analysis of the striatum revealed a significant cluster of activation that encompassed regions of the left caudate, putamen and nucleus accumbens (Figure 4; Table 2, lower part). These activated areas have been associated with reward processing, the coding of "reward-prediction errors" (i.e., the difference between actual and expected reward) and reinforcement learning (O'Doherty, 2004; Daw and Doya, 2006; Haruno and Kawato, 2006). Since the uncertainty induced by a blurred picture was relieved by the following picture on only half of the trials, the reduction of perceptual uncertainty by the second picture possibly caused a (partial) reward-prediction error. Accordingly, the striatal activation could reflect the reward value and/or the reward-prediction error associated with the relief of perceptual uncertainty.

Confirming predictions, the ROI analysis of the hippocampus revealed that regions of the bilateral hippocampus showed stronger activation in response to the second picture in the

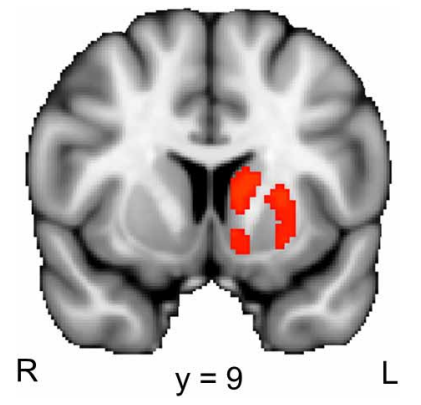

FIGURE 4 | Striatal activation associated with the reduction of perceptual uncertainty. The colored regions were more active when the second picture in a trial reduced the perceptual uncertainty induced by the preceding picture (i.e., the second picture in the $\mathrm{B}-\mathrm{C}_{\text {corresponding }}$ condition) than when the second picture did not reduce the perceptual uncertainty induced by the preceding picture (i.e., the second picture in the $\mathrm{B}-\mathrm{C}_{\text {unrelated }}$ condition). $R$, right; $L$, left. The displayed activations are the cluster-corrected $Z$ statistic maps $(p<0.05$ ) from the striatal $\mathrm{ROI}$ analysis, which were overlaid onto the standard $\mathrm{MNI}$ brain.

$\mathrm{B}-\mathrm{C}_{\text {corresponding }}$ than in the $\mathrm{B}-\mathrm{C}_{\text {unrelated }}$ condition (Figure 5). A contrast that identified brain regions where activation was larger in response to the second picture in the $\mathrm{B}-\mathrm{C}_{\text {corresponding }}$ condition than in the $\mathrm{C}-\mathrm{C}$ condition also revealed significant activation in the bilateral hippocampus (512 and $88 \mathrm{~mm}^{3}$ in the left and right hippocampus, respectively). The event-related time courses of the BOLD signal in response to the second picture in each of the four conditions illustrate the specific increase in hippocampal activation for the $\mathrm{B}-\mathrm{C}_{\text {corresponding }}$ condition (Figure 5). The increased hippocampal activation in response to uncertaintyreducing stimuli likely underlied the enhanced later recall of these stimuli. Interestingly, the strength of participants' hippocampal activation in response to the reduction of uncertainty was positively correlated with the strength of their AIC activation in response to the induction of uncertainty $(r=0.57, p=0.01$ and $r=0.53, p=0.02$ for the left and the right hippocampus, respectively).

The whole-brain analysis for this contrast also revealed striatal activation, as well as activation of the lateral occipital cortex, orbitofrontal cortex and posterior insula (Table 3 ).

\section{DISCUSSION}

The present results provide the first demonstration of the neurobiological basis of human perceptual curiosity. By elucidating the neural underpinnings of the induction and relief of perceptual curiosity, our study extends existing behavioral accounts of curiosity. In particular, our results are consistent with Berlyne's classic psychological theory of curiosity (Berlyne, 1954, 1960, 1966). First, our finding that perceptual uncertainty activated brain regions sensitive to arousal and conflict supports the assumption that curiosity evoked by ambiguous stimuli is an aversive condition, and induces an increase in arousal. Second, our finding that the reduction of perceptual uncertainty activated striatal regions involved in reward processing supports the assumption that the termination of this condition, through access to relevant information, is rewarding. Third, our findings 

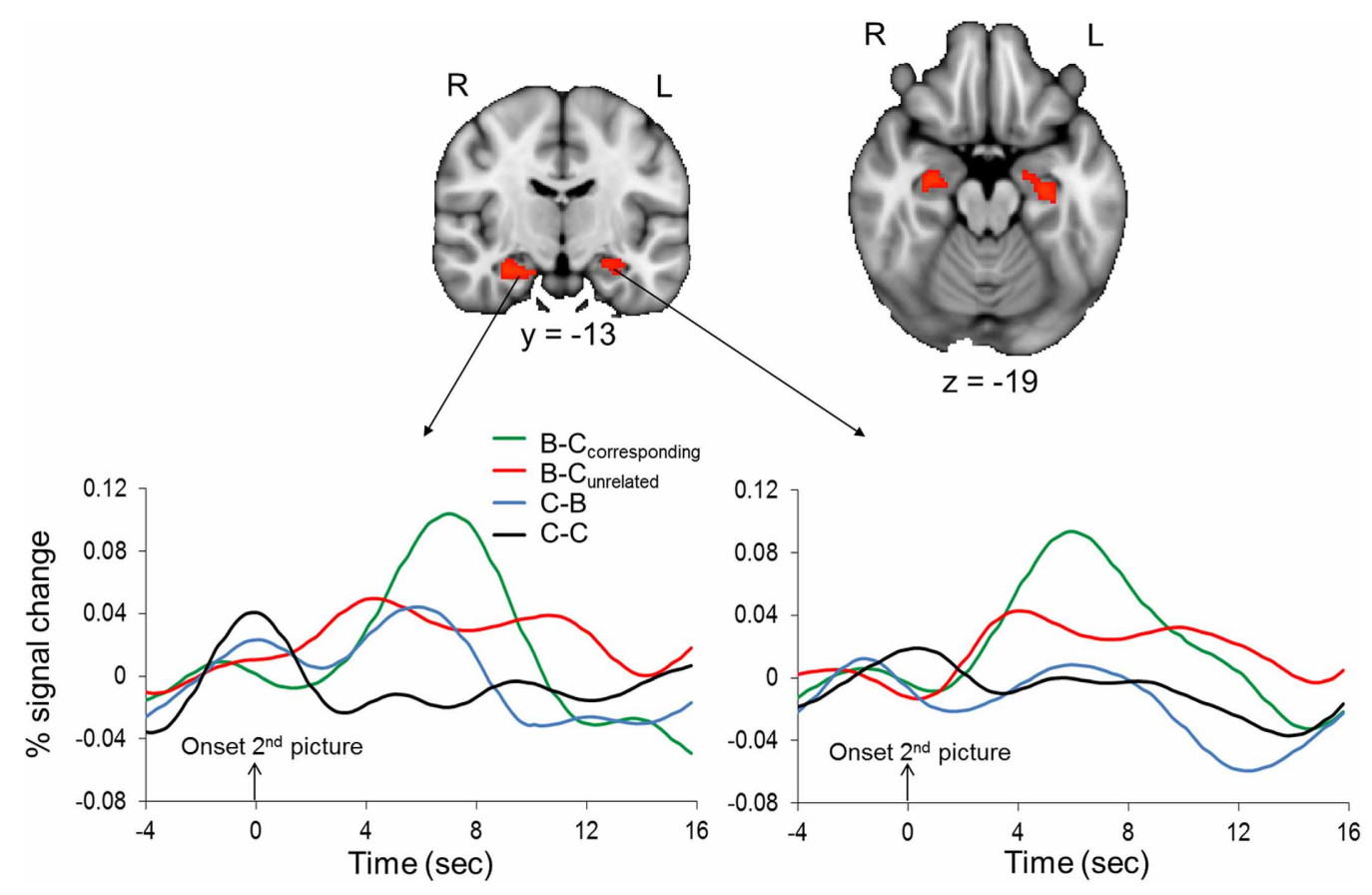

FIGURE 5 | Hippocampal activation associated with the reduction of perceptual uncertainty. Upper panel: the colored regions were more active when the second picture in a trial reduced the perceptual uncertainty induced by the preceding picture (i.e., the second picture in the $\mathrm{B}-\mathrm{C}_{\text {corresponding }}$ condition) than when the second picture did not reduce the perceptual uncertainty induced by the preceding picture (i.e., the second picture in the
B- $\mathrm{C}_{\text {unrelated }}$ condition). The displayed activations are the cluster-corrected $Z$ statistic maps from the hippocampal ROI analysis $(p<0.05)$ overlaid onto the standard MNI brain. Lower panel: Time course of hemodynamic activity in response to the second picture in each of the four conditions. Time courses were extracted from the hippocampal activation clusters shown in the upper panel. that the reduction of perceptual uncertainty was associated with increased hippocampal activation and enhanced incidental memory support the assumption that uncertainty reduction facilitates memory and learning.

Our findings are also consistent with Loewenstein's information-gap account of curiosity which proposes that curiosity is a negative feeling of deprivation that is caused by an inconsistency, or gap, between one's actual and aspired level of knowledge (Loewenstein, 1994; see Litman et al., 2005, for empirical tests and a more detailed investigation of this theory). Since people differ in their aspired level of knowledge, the same actual level of knowledge will evoke curiosity in some people but not in others. In line with this idea, we found that inter-individual variation in trait perceptual curiosity correlated with the strength of AIC activation in response to perceptual uncertainty, suggesting that people with a higher level of aspired perceptual knowledge experience stronger negative feelings when confronted with ambiguous perceptual input.

The relief of perceptual curiosity was associated with activation in regions of the striatum (left caudate, putamen and nucleus accumbens) involved in reward processing, which is consistent with the idea that curiosity reduction is rewarding. This idea is consistent with previous behavioral findings that people actively prefer to view the clear version of a preceding blurred picture over viewing an unrelated clear picture (Nicki, 1970). Other work has shown that people have a similar preference for exploring perceptually novel over familiar stimuli, a tendency that is also associated with striatal activation (Wittmann et al., 2008). In the reinforcement-learning literature, this bias towards the exploration of uncertain or novel options is captured by the concept of an "exploration bonus" that is assigned to uncertain or novel stimuli to promote their exploration (Kakade and Dayan, 2002).

The relief of perceptual curiosity was also associated with enhanced incidental memory, and with increased hippocampal activation, a plausible neural substrate underlying the behavioral memory effect. The finding that curiosity reduction leads to enhanced memory suggests that inducing people's curiosity before presenting them with teaching material (e.g., by asking people to guess the meaning of foreign words before showing them the translations) can facilitate learning.

Because the AIC and ACC are the two brain regions that are typically activated by aversive conditions, and the striatum plays a key role in reward processing, our fMRI results are consistent with our predictions that curiosity is an aversive state whose termination is rewarding. However, it is important to note that the $\mathrm{AIC/ACC}$ and the striatum are also involved in cognitive processes that are unrelated to aversive states and rewards, respectively. Due to the non-specificity of the function of these brain areas, it is possible that the activations we found reflected processes other than those predicted by Berlyne's theory; hence our findings do not provide persuasive evidence for that theory. Indeed, our method of inferring the presence of particular cognitive/affective processes from the activation of particular brain regions is an example of "reverse inference" (Poldrack, 2006). Although 
this method is not deductively valid, it can provide interesting hypotheses about the underlying mechanisms of relatively unexplored conditions, such as curiosity, which can then be tested in subsequent studies. Thus, our study should be seen as a first demonstration of the neural correlates of perceptual curiosity, of which the results are consistent with but do not provide unequivocal evidence for Berlyne's theory. Future studies testing more specific predictions are necessary to either confirm or reject our interpretation of the curiosity-related brain activation.

Curiosity is a multifaceted construct, and several different types of curiosity can be distinguished. One important distinction is the difference between perceptual and epistemic curiosity. Perceptual curiosity is aroused by novel, strange or ambiguous stimuli, whereas epistemic curiosity refers to the desire for knowledge or intellectual information which applies mainly to humans (Berlyne, 1954). Another, orthogonal, distinction can be made between specific and diversive curiosity, referring to the desire for a particular piece of information versus the more general stimulation-seeking motive that is closely related to boredom (Berlyne, 1960; see Litman, 2008, for a related distinction). The type of curiosity that we investigated in the present study can be referred to as specific perceptual curiosity, one of the most basic types of curiosity that applies to both animals and humans. The neural substrates of specific epistemic curiosity evoked by trivia questions was investigated recently (Kang et al., 2009). Kang et al. found that questions that were rated as more puzzling were associated with stronger activation in regions of the caudate. However, since the questions were always followed by their correct answers, it was unclear whether this activation reflected uncertainty about the correct answer, feedback anticipation, or a combination of the two. In our study, the uncertainty induced by blurred pictures was often not relieved, which allowed examination of the neural correlates of pure curiosity unconfounded by the anticipation of uncertainty-reducing feedback. In addition, by comparing conditions in which the second picture did vs. did not reduce perceptual uncertainty, we could separately isolate the neural correlates of the relief of curiosity.

We did not ask participants to rate their curiosity on each trial since we were concerned that this would confound the brain activation reflecting their natural curiosity. Therefore, a limitation of our study is that we could not take into account trial-to-trial

\section{REFERENCES}

Beckmann, C. F., Jenkinson, M., and Smith, S. M. (2003). General multilevel linear modeling for group analysis in FMRI. Neuroimage 20, 1052-1063.

Berlyne, D. E. (1954). A theory of human curiosity. Br. J. Psychol. 45, 180-191.

Berlyne, D. E. (1960). Conflict, Arousal and Curiosity. New York, NY: McGraw-Hill.

Berlyne, D. E. (1965). Structure and Direction in Thinking. New York, NY: John Wiley.

Berlyne, D. E. (1966). Curiosity and exploration. Science 153, 25-33.
Berlyne, D. E., and Borsa, D. M. (1968). Uncertainty and the orientation reaction. Percept. Psychophys. 3, 77-79.

Berlyne, D. E., and Normore, L. F. (1972). Effects of prior uncertainty on incidental free recall. J. Exp. Psychol. 96, 43-48.

Collins, R. P., Litman, J. A., and Spielberger, C. D. (2004). The measurement of perceptual curiosity. Pers. Individ. Dif. 36, 1127-1141.

Craig, A. D., Reiman, E. M., Evans, A., and Bushnell, M. C. (1996). Functional imaging of an illusion of pain. Nature 384, 258-260.

variation in experienced curiosity. In addition, it must be noted that the blurred pictures in our study were associated with both perceptual uncertainty and outcome uncertainty, since the identity of the blurred image was revealed in only half of the trials. Thus, participants may have experienced uncertainty not only about the identity of the image, but also about whether or not the identity of the image would be resolved. Both forms of uncertainty are likely to increase overall curiosity, but future studies in which these two forms of uncertainty are manipulated independently are needed to assess their respective contributions to brain activation. Finally, it is likely that curiosity reduction through passive exposure to uncertainty-reducing stimuli, as examined in the present study, differs from curiosity reduction that is achieved through active exploration. A recent study showed that hippocampus activation was stronger when people had volitional control over the visual exploration of pictures in a visual-learning task than when they received exactly the same visual information in a passive condition (Voss et al., 2011). This suggests that the hippocampus activation associated with uncertainty reduction that we found in the present study would have been even stronger if participants would have had the opportunity to actively control the exploration of uncertainty-inducing stimuli.

To conclude, our results provide the first demonstration of the neural correlates of human perceptual curiosity, and are consistent with the assumptions of Berlyne's theory that perceptual curiosity evokes an aversive state of increased arousal, whose termination is rewarding and promotes incidental memory. Because curiosity plays a key role in many aspects of human behavior, a better understanding of the psychological and neurobiological basis of curiosity may have considerable practical implications for various societal objectives. Together with previous behavioral findings (Berlyne and Normore, 1972), our results suggest that inventing ways to arouse people's curiosity could contribute to the optimization of educational systems and advertising strategies, and may promote scientific discovery.

\section{ACKNOWLEDGMENTS}

This research was supported by VIDI grants from the Netherlands Organization for Scientific research to Sander Nieuwenhuis and Serge A. R. B. Rombouts. We thank Stephen Brown and Eefje Zeestraten for scanning assistance.

Critchley, H. D. (2005). Neural mechanisms of autonomic, affective, and cognitive integration. J. Comp. Neurol. 493, 154-166.

Daw, N. D., and Doya, K. (2006). The computational neurobiology of learning and reward. Curr. Opin. Neurobiol. 16, 199-204.

Eisenberger, N. I., Lieberman, M. D., and Williams, K. D. (2003). Does rejection hurt? An FMRI study of social exclusion. Science 302, 290-292.

Fornito, A., Whittle, S., Wood, S. J., Velakoulis, D., Pantelis, C., and Yücel, M. (2006). The influence of sulcal variability on morphometry of the human anterior cingulate and paracingulate cortex. Neuroimage 33, 843-854.

Haruno, M., and Kawato, M. (2006). Different neural correlates of reward expectation and reward expectation error in the putamen and caudate nucleus during stimulus-action-reward association learning. J. Neurophysiol. 95, 948-959.

Jenkinson, M., Bannister, P., Brady, M., and Smith, S. M. (2002). Improved optimization for the robust and accurate linear registration and motion correction of brain images. Neuroimage 17, 825-841. 
Jenkinson, M., and Smith, S. M. (2001). A global optimisation method for robust affine registration of brain images. Med. Image Anal. 5, 143-156.

Kakade, S., and Dayan, P. (2002). Dopamine: generalization and bonuses. Neural Netw. 15, 549-559.

Kang, M. J., Hsu, M., Krajbich, I. M., Loewenstein, G., McClure, S. M., Wang, J. T., and Camerer, C. F. (2009). The wick in the candle of learning: epistemic curiosity activates reward circuitry and enhances memory. Psychol. Sci. 20, 963-973.

Litman, J. A. (2008). Interest and deprivation factors of epistemic curiosity. Pers. Individ. Dif. 44, 1585-1595.

Litman, J. A., Hutchins, T. L., and Russon, R. K. (2005). Epistemic curiosity, feeling-of-knowing, and exploratory behaviour. Cogn. Emot. 19, 559-582.

Loewenstein, G. (1994). The psychology of curiosity: a review and reinterpretation. Psychol. Bull. 116, 75-98.

Nicki, R. M. (1970). The reinforcing effect of uncertainty reduction on a human operant. Can. J. Psychol. 24, 389-399.

O’Doherty, J. P. (2004). Reward representations and reward-related learning in the human brain: insights from neuroimaging. Curr. Opin. Neurobiol. 14, 769-776.

Peyron, R., Laurent, B., and GarciaLarrea, L. (2000). Functional imaging of brain responses to pain. A review, meta-analysis. Neurophysiol. Clin. 30, 263-288.

Ploghaus, A., Tracey, I., Gati, J. S., Clare, S., Menon, R. S., Matthews, P. M., and Rawlins, J. N. (1999). Dissociating pain from its anticipation in the human brain. Science 284, 1979-1981.

Poldrack, R. A. (2006). Can cognitive processes be inferred from neuroimaging data? Trends Cogn. Sci. 10, 59-63.

Raichle, M. E., MacLeod, A. M., Snyder, A. Z., Powers, W. J., Gusnard, D. A., and Shulman, G. L. (2001). A default mode of brain function. Proc. Natl. Acad. Sci. U.S.A.98, 676-682.

Ridderinkhof, K. R., Ullsperger, M., Crone, E. A., and Nieuwenhuis, S. (2004). The role of medial frontal cortex in cognitive control. Science 306, 443-447.

Rossion, B., and Pourtois, G. (2004). Revisiting Snodgrass and Vanderwart's object pictorial set: the role of surface detail in basic-level object recognition. Perception 33, 217-236.

Seeley, W. W., Menon, V., Schatzberg, A. F., Keller, J., Glover, G. H., Kenna, H., Reiss, A. L., and Greicius, M. D. (2007). Dissociable intrinsic connectivity networks for salience processing and executive control. J. Neurosci. 27, 2349-2356.

Shackman, A. J., Salomons, T. V., Slagter, H. A., Fox, A. S., Winter, J. J., and Davidson, R. J. (2011). The integration of negative affect, pain and cognitive control in the cingulate cortex. Nat. Rev. Neurosci. 12, 154-167.
Shannon, C. E. (1948). A mathematical theory of communication. Bell Syst. Tech. J. 27, 379-423, 623-656.

Singer, T., Critchley, H. D., and Preuschoff, K. (2009). A common role of insula in feelings, empathy and uncertainty. Trends Cogn. Sci. 13, 334-340.

Smith, S. M. (2002). Fast robust automated brain extraction. Hum. Brain Mapp. 17, 143-155.

Smith, S. M., Jenkinson, M., Woolrich, M. W., Beckmann, C. F., Behrens, T. E., Johansen-Berg, H., Bannister, P. R., De Luca, M., Drobnjak, I., Flitney, D. E., Niazy, R. K., Saunders, J., Vickers, J., Zhang, Y. De Stefano, N., Brady, J. M., and Matthews, P. M. (2004). Advances in functional and structural MR image analysis and implementation as FSL. Neuroimage 23(Suppl. 1), S208-S219.

Ullsperger, M., Harsay, H. A., Wessel, J. R., and Ridderinkhof, K. R. (2010) Conscious perception of errors and its relation to the anterior insula. Brain Struct. Funct. 214, 629-643.

Voss, J. L., Gonsalves, B. D., Federmeier, K. D., Tranel, D., and Cohen, N. J. (2011). Hippocampal brainnetwork coordination during volitional exploratory behavior enhances learning. Nat. Neurosci. 14, 115-120.

Wittmann, B. C., Daw, N. D., Seymour, B., and Dolan, R. J. (2008). Striatal activity underlies novelty-based choice in humans. Neuron 58, 967-973.

Woolrich, M. W., Behrens, T. E., Beckmann, C. F., Jenkinson, M. and Smith, S. M. (2004). Multilevel linear modelling for FMRI group analysis using Bayesian inference. Neuroimage 21, 1732-1747.

Woolrich, M. W., Ripley, B. D., Brady, M., and Smith, S. M. (2001). Temporal autocorrelation in univariate linear modeling of FMRI data. Neuroimage 14, 1370-1386.

Worsley, K. J. (2001) "Statistical analysis of activation images," in Functional MRI: An Introduction to Methods, eds P. M. Jezzard, P. M. Matthews, and S. M. Smith (Oxford: Oxford University Press), 251-270.

Conflict of Interest Statement: The authors declare that the research was conducted in the absence of any commercial or financial relationships that could be construed as a potential conflict of interest.

Received: 28 June 2011; accepted: 30 January 2012; published online: 13 February 2012.

Citation: Jepma $M$, Verdonschot $R G$, van Steenbergen $H$, Rombouts $S A R B$ and Nieuwenhuis $S$ (2012) Neural mechanisms underlying the induction and relief of perceptual curiosity. Front. Behav. Neurosci. 6:5. doi: 10.3389/fnbeh. 2012.00005

Copyright (c) 2012 Jepma, Verdonschot, van Steenbergen, Rombouts and Nieuwenhuis. This is an open-access article distributed under the terms of the Creative Commons Attribution Non Commercial License, which permits non-commercial use, distribution, and reproduction in other forums, provided the original authors and source are credited. 